\title{
The Role of H-Bonding in Non-Concerted Proton-Coupled Electron Transfer: Explaining the Voltammetry of Phenylenediamines in the Presence of Weak
}

\section{Bases in Acetonitrile}

\author{
Laurie A. Clare, Tammy D. Pham, Lily A. Rafou, Ayla G. Buenaventura, Tiffany R. Scott, \\ Veronika Mikhaylova and Diane K. Smith* \\ Department of Chemistry and Biochemistry, San Diego State University \\ San Diego, CA 92182-1030
}

\section{SUPPORTING INFORMATION}

\section{TABLE OF CONTENTS}

Figure S1. UV-Vis of fully oxidized (quinoidal form) of $0.5 \mathrm{mM} \mathrm{H}_{2} \mathrm{PD}$ in different protonation

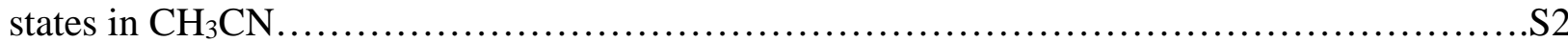

Figure S2. UV-Vis of 1:1 $\mathrm{H}_{2} \mathrm{PD}$ :pyridine in different $\mathrm{H}_{2} \mathrm{PD}$ oxidation states in $\mathrm{CH}_{3} \mathrm{CN}$.......S2 Figure S3. CV's of $0.5 \mathrm{mM} \mathrm{H}_{2} \mathrm{PD}$ in $\mathrm{CH}_{3} \mathrm{CN}$ at $0.2 \mathrm{~V} / \mathrm{s}$ with $0.25,0.50,0.75,1.0,2.0$ and 4.0 eq

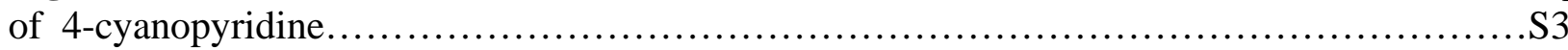

Figure S4. (a) CV's of $0.5 \mathrm{mM} \mathrm{H} \mathrm{H}_{2} \mathrm{PD}$ in $\mathrm{CH}_{3} \mathrm{CN}$ at $0.2 \mathrm{~V} / \mathrm{s}$ with increasing amounts of 4-

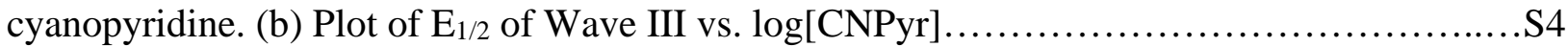
Figure S5. (a) CV's of $0.5 \mathrm{mM} \mathrm{H} \mathrm{H}_{2} \mathrm{PD}$ in $\mathrm{CH}_{3} \mathrm{CN}$ at $0.2 \mathrm{~V} / \mathrm{s}$ with increasing amounts of 4-

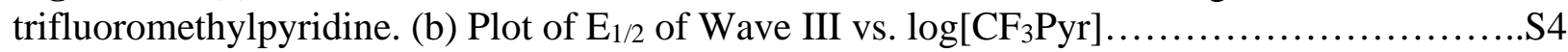
Figure S6. (a) CV's of $0.5 \mathrm{mM} \mathrm{H} \mathrm{H}_{2} \mathrm{PD}$ in $\mathrm{CH}_{3} \mathrm{CN}$ at $0.2 \mathrm{~V} / \mathrm{s}$ with increasing amounts of 4-

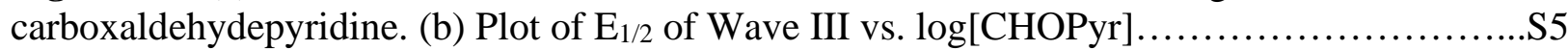
Figure S7. (a) CV's of $0.5 \mathrm{mM} \mathrm{H}_{2} \mathrm{PD}$ in $0.1 \mathrm{M} \mathrm{CH}_{3} \mathrm{CN}$ at $0.2 \mathrm{~V} / \mathrm{s}$ with .......increasing amounts

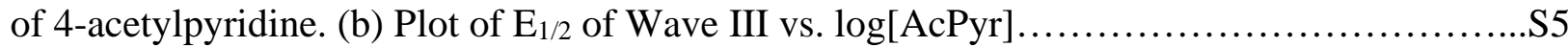
Table S1. Ave. E1/2's for Wave III (V vs Fc) with 0.5 mM H$~_{2} \mathrm{PD} / 0.5 \mathrm{mM}$ Pyridine Derivatives. (Data plotted in Figure 6.).................................................... 6 Table S2. Mechanism and parameters used for simulating CVs in Figure 7a (H-Bonding square

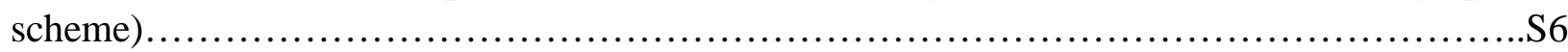

Table S3. Mechanism and parameters ${ }^{1}$ used for simulating CVs in Figure $7 \mathrm{~b}$ (Proton Transfer square scheme).

Table S4. Mechanism and parameters used for simulating CVs in Figure 8a (Concerted ProtonElectron Transfer square scheme).

Table S5. Mechanism and parameters used for simulating CVs in Figure 8b (Wedge scheme)..S7

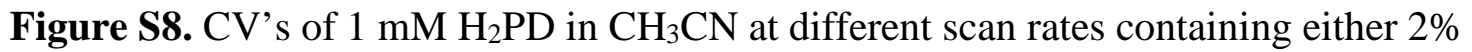

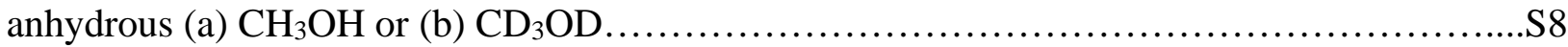
Figure S9. CV's of $1 \mathrm{mM} \mathrm{H} \mathrm{H}_{2} \mathrm{PD}+10 \mathrm{mM}$ 4-cyanopyridine in $\mathrm{CH}_{3} \mathrm{CN}$ at different scan rates

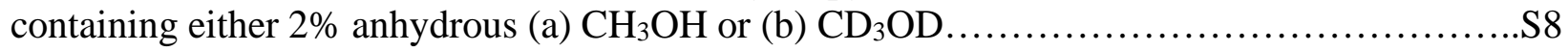
Figure S10. CV's of $1 \mathrm{mM} \mathrm{H} \mathrm{H}_{2} \mathrm{PD}$ in $\mathrm{CH}_{3} \mathrm{CN}$ at (a) different scan rates with $9.6 \mathrm{mM} 4$ cyanopyridine, and (b) at $1 \mathrm{~V} / \mathrm{s}$ with different concentrations of 4-cyanopyridine.... 


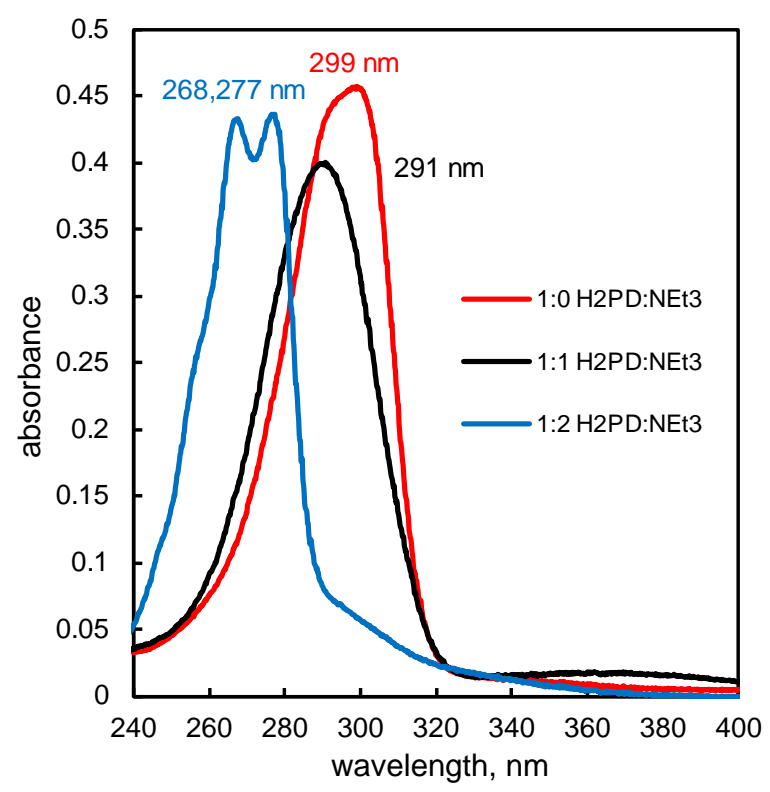

Figure S1. UV-Vis of fully oxidized (quinoidal form) of $0.5 \mathrm{mM} \mathrm{H}_{2} \mathrm{PD}$ in different protonation states in $0.1 \mathrm{M} \mathrm{NBu}_{4} \mathrm{PF}_{6} / \mathrm{CH}_{3} \mathrm{CN}$ : (red trace) $\mathrm{H}_{2} \mathrm{PD}^{2+}$; (black trace) $\mathrm{HPD}^{+}$; (blue spectrum) PD. The spectra were generated in a thin-layer spectroelectrochemical cell by holding the potential positive of the second oxidation of $\mathrm{H}_{2} \mathrm{PD}$ in the presence of 0,1 and 2 equivalents of triethylamine, respectively.

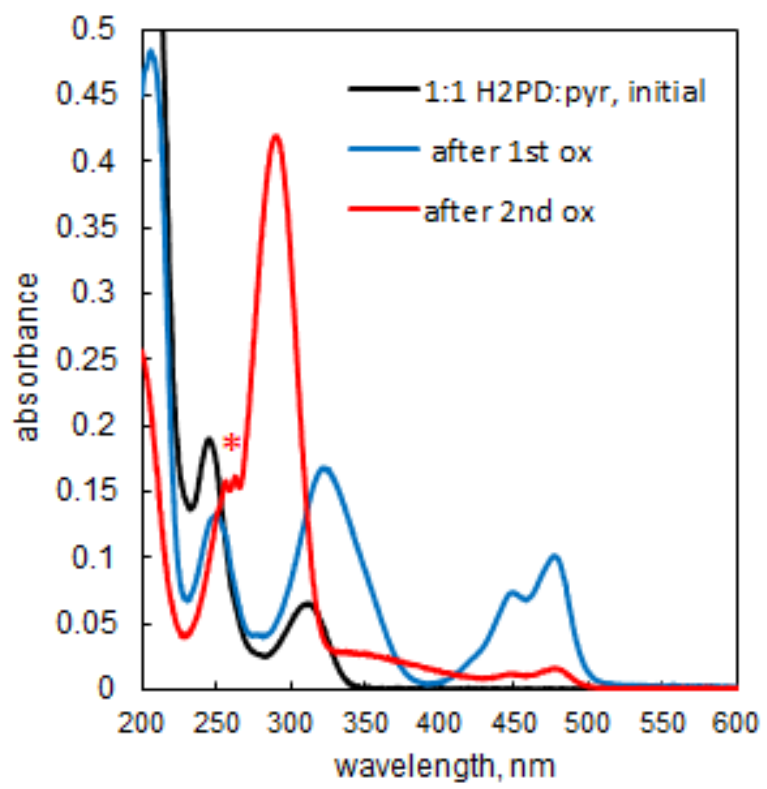

Figure S2. UV-Vis spectra taken in a thin layer spectroelectrochemical cell of $0.5 \mathrm{mM} \mathrm{H}_{2} \mathrm{PD}+$ $0.5 \mathrm{mM}$ pyridine in $0.1 \mathrm{M} \mathrm{NBu}_{4} \mathrm{PF}_{6} / \mathrm{CH}_{3} \mathrm{CN}$ : (black spectrum) initial solution; (blue spectrum) electrode held at the potential of the first oxidation wave of $\mathrm{H}_{2} \mathrm{PD}$; (red spectrum) electrode held at a potential positive of the second oxidation of $\mathrm{H}_{2} \mathrm{PD}$. Note that the characteristic absorption peak of pyridinium (indicated with an asterisk) is only observed at the second oxidation, indicating that proton transfer to make pyridinium only occurs at the second oxidation. 


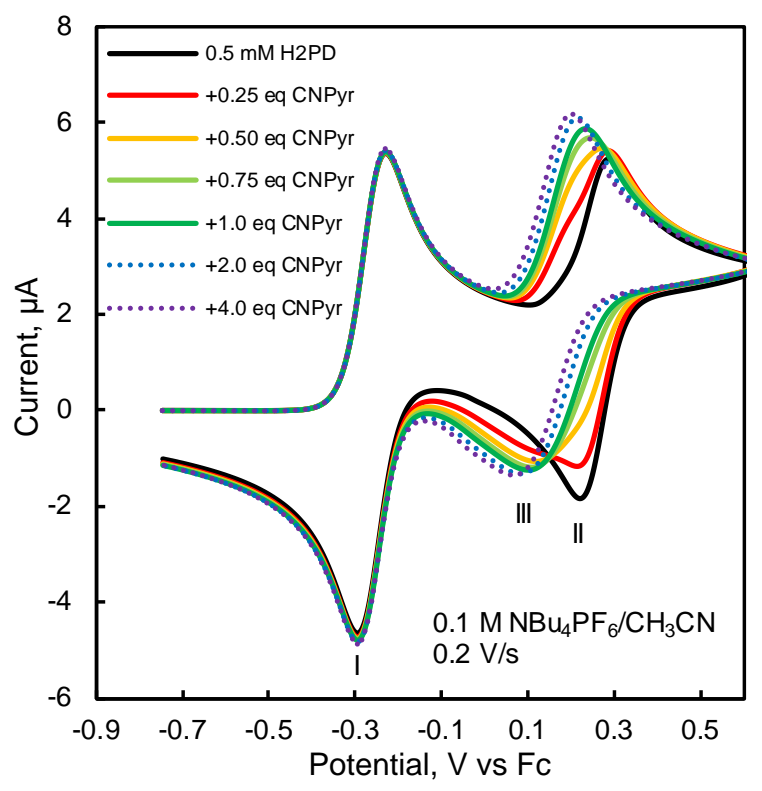

Figure S3. Background-subtracted CV's of $0.5 \mathrm{mM} \mathrm{H}_{2} \mathrm{PD}$ in $0.1 \mathrm{M} \mathrm{NBu} 4 \mathrm{PF}_{6} / \mathrm{CH}_{3} \mathrm{CN}$ at $0.2 \mathrm{~V} / \mathrm{s}$ with $0.25,0.50,0.75,1.0,2.0$ and 4.0 eq of 4 -cyanopyridine. 

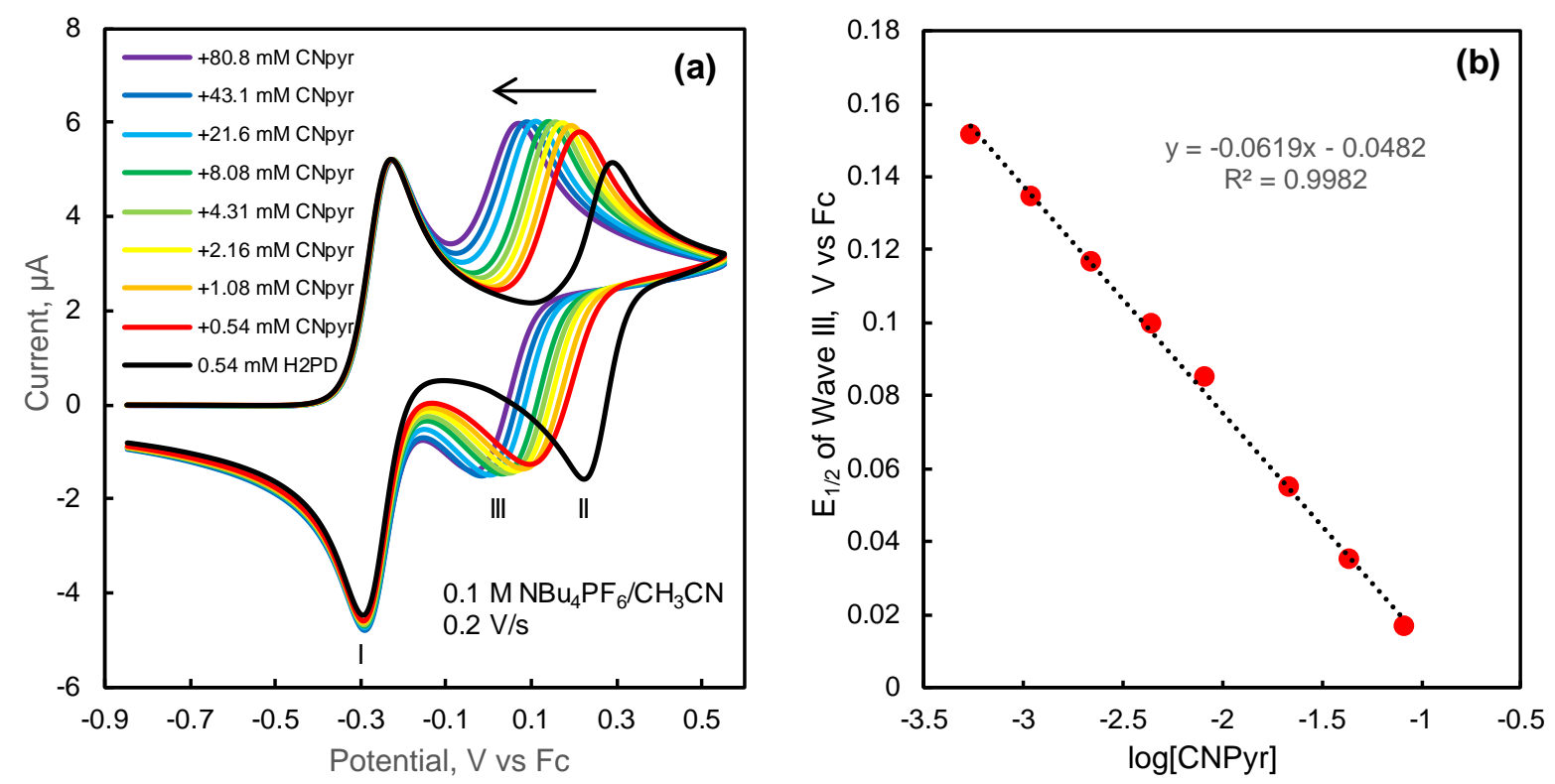

Figure S4. (a) Background-subtracted CV's of $0.5 \mathrm{mM} \mathrm{H}_{2} \mathrm{PD}$ in $0.1 \mathrm{M} \mathrm{NBu}_{4} \mathrm{PF}_{6} / \mathrm{CH}_{3} \mathrm{CN}$ at 0.2 $\mathrm{V} / \mathrm{s}$ with increasing amounts of 4-cyanopyridine. (b) Plot of $\mathrm{E}_{1 / 2}$ of Wave III vs. log[CNPyr].
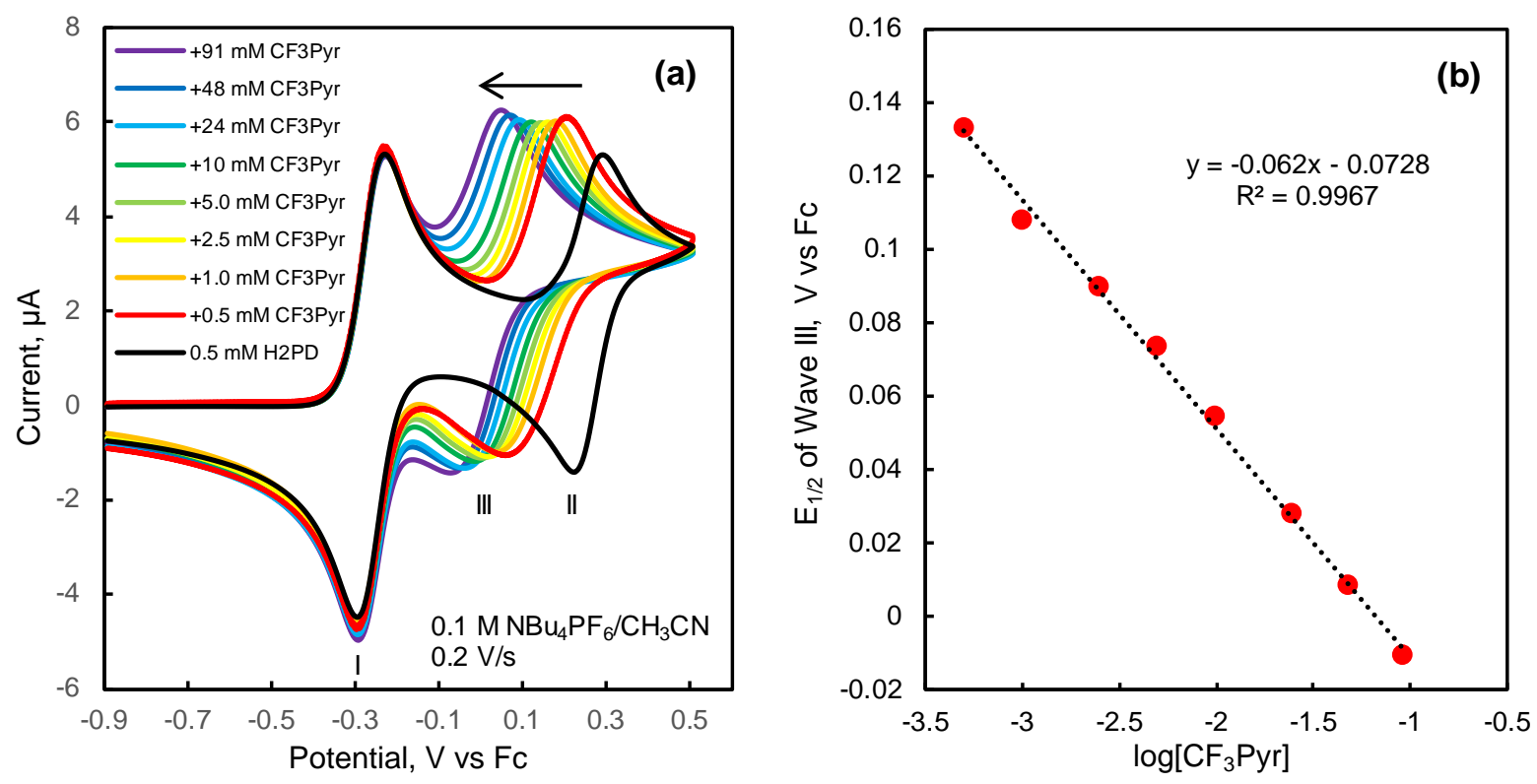

Figure S5. (a) Background-subtracted CV's of $0.5 \mathrm{mM} \mathrm{H}_{2} \mathrm{PD}$ in $0.1 \mathrm{M} \mathrm{NBu}_{4} \mathrm{PF}_{6} / \mathrm{CH}_{3} \mathrm{CN}$ at 0.2 $\mathrm{V} / \mathrm{s}$ with increasing amounts of 4-trifluoromethylpyridine. (b) Plot of $\mathrm{E}_{1 / 2}$ of Wave III vs. $\log \left[\mathrm{CF}_{3} \mathrm{Pyr}\right]$. 

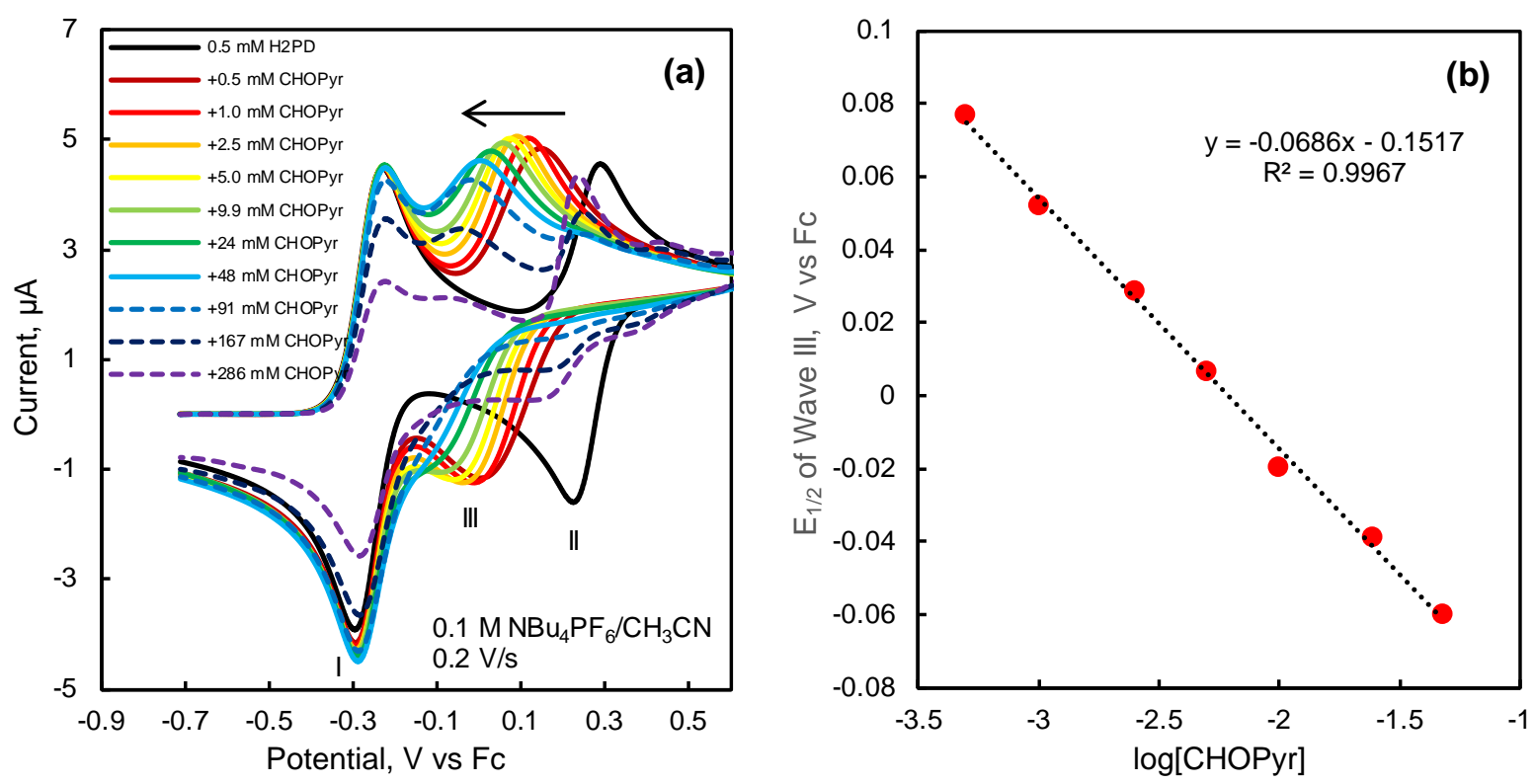

Figure S6. (a) Background-subtracted CV's of $0.5 \mathrm{mM} \mathrm{H}_{2} \mathrm{PD}$ in $0.1 \mathrm{M} \mathrm{NBu}_{4} \mathrm{PF}_{6} / \mathrm{CH}_{3} \mathrm{CN}$ at 0.2 V/s with increasing amounts of 4-carboxaldehydepyridine. (b) Plot of $\mathrm{E}_{1 / 2}$ of Wave III vs. $\log [\mathrm{CHOPyr}]$.
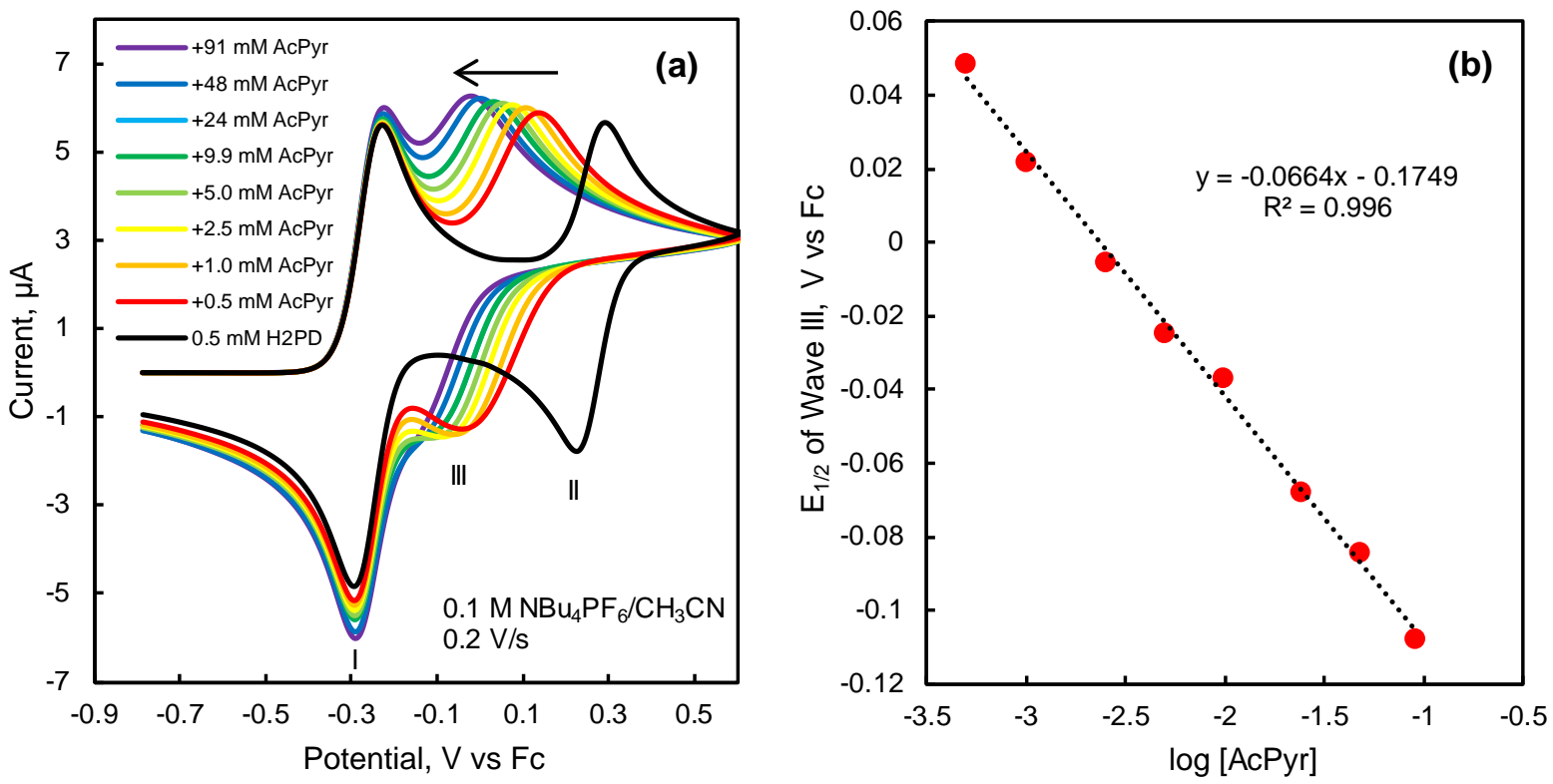

Figure S7. (a) Background-subtracted CV's of $0.5 \mathrm{mM} \mathrm{H}_{2} \mathrm{PD}$ in $0.1 \mathrm{M} \mathrm{NBu}_{4} \mathrm{PF}_{6} / \mathrm{CH}_{3} \mathrm{CN}$ at 0.2 $\mathrm{V} / \mathrm{s}$ with increasing amounts of 4-acetylpyridine. (b) Plot of $\mathrm{E}_{1 / 2}$ of Wave III vs. log[AcPyr]. 
Table S1. Ave. E1/2's for Wave III (V vs Fc) with $0.5 \mathrm{mM} \mathrm{H}_{2} \mathrm{PD} / 0.5 \mathrm{mM}$ Pyridine Derivatives. (Data plotted in Figure 6.)

\begin{tabular}{|l|c|c|c|c|c|c|}
\hline $\begin{array}{c}\text { Pyridine } \\
\text { 4-Substituent }\end{array}$ & $\begin{array}{c}\mathbf{p K}_{\mathbf{a}}\left(\mathbf{H}_{\mathbf{2}} \mathbf{O}\right) \\
\text { of HPyr }^{+}\end{array}$ & $\begin{array}{c}\mathbf{p K}_{\mathbf{a}} \\
\text { St Dev }\end{array}$ & $\begin{array}{c}\text { Ave. } \mathbf{E}_{\mathbf{1} / \mathbf{2}} \\
\text { Wave III }\end{array}$ & $\begin{array}{c}\mathbf{E}_{\mathbf{1} / \mathbf{2}} \\
\text { St Dev }\end{array}$ & $\begin{array}{c}\mathbf{E}_{\mathbf{1} / \mathbf{2}} \\
\mathbf{9 5 \%} \mathbf{C} \text {. L. }\end{array}$ & $\begin{array}{c}\text { Sample } \\
\text { Size }\end{array}$ \\
\hline$-\mathrm{CN}$ & 1.92 & 0.1 & 0.162 & 0.0076 & 0.0080 & 6 \\
\hline$-\mathrm{CF}_{3}$ & 2.92 & 0.1 & 0.133 & 0.0129 & 0.0319 & 3 \\
\hline$-\mathrm{C}(\mathrm{O}) \mathrm{H}$ & 3.3 & 0.1 & 0.092 & 0.008 & 0.0202 & 3 \\
\hline$-\mathrm{C}(\mathrm{O}) \mathrm{CH}_{3}$ & 3.57 & 0.1 & 0.045 & 0.0092 & 0.0147 & 4 \\
\hline$-\mathrm{H}$ & 5.23 & 0.1 & -0.048 & 0.0018 & 0.0028 & 4 \\
\hline
\end{tabular}

Table S2. Mechanism and parameters ${ }^{1}$ used for simulating CVs in Figure 7a (H-Bonding square scheme).

\begin{tabular}{|l|l|l|l|}
\hline & Reaction & $\mathbf{E}^{\circ}$ or $\mathbf{K}^{2}$ & $\mathbf{k}^{\circ}$ or kf \\
\hline E1 & $\mathrm{H}_{2} \mathrm{PD}^{+}+\mathrm{e}=\mathrm{H}_{2} \mathrm{PD}$ & $-0.262 \mathrm{~V}$ & $10 \mathrm{~cm} / \mathrm{s}$ \\
\hline E2 & $\mathrm{H}_{2} \mathrm{PD}^{2+}+\mathrm{e}=\mathrm{H}_{2} \mathrm{PD}^{+}$ & $+0.256 \mathrm{~V}$ & $10 \mathrm{~cm} / \mathrm{s}$ \\
\hline E4 & $\mathrm{BH}_{2} \mathrm{PD}^{2+}+\mathrm{e}=\mathrm{BH}_{2} \mathrm{PD}^{+}$ & $-0.362 \mathrm{~V}$ & $10 \mathrm{~cm} / \mathrm{s}$ \\
\hline HB1 & $\mathrm{H}_{2} \mathrm{PD}^{+}+\mathrm{B}=\mathrm{BH}_{2} \mathrm{PD}^{+}$ & $\left(1.90 \times 10^{-9} \mathrm{M}^{-1}\right)=\mathrm{K}_{\mathrm{rad}}$ & $1.90 \mathrm{M}^{-1} \mathrm{~s}^{-1}$ \\
\hline $\mathrm{HB} 2$ & $\mathrm{H}_{2} \mathrm{PD}^{2+}+\mathrm{B}=\mathrm{BH}_{2} \mathrm{PD}^{2+}$ & $52.8 \mathrm{M}^{-1}=\mathrm{K}_{\mathrm{ox}}$ & $1.0 \times 10^{9} \mathrm{M}^{-1} \mathrm{~s}^{-1}$ \\
\hline
\end{tabular}

${ }^{1} \alpha=0.5$ for all heterogeneous electron transfers; electrode area $=0.0206 \mathrm{~cm}^{2} ; \mathrm{Ru}=336 \Omega$; D of all species $=$ $1.45 \times 10^{5} \mathrm{~cm}^{2} \mathrm{~s}^{-1}$.

${ }^{2}$ Values in parentheses are automatically set by other thermodynamic parameters.

Table S3. Mechanism and parameters ${ }^{1}$ used for simulating CVs in Figure $7 \mathrm{~b}$ (Proton Transfer square scheme).

\begin{tabular}{|l|l|l|l|}
\hline & Reaction & $\mathbf{E}^{\circ}$ or $\mathbf{K}^{2}$ & $\mathbf{k}^{\circ}$ or kf \\
\hline E1 & $\mathrm{H}_{2} \mathrm{PD}^{+}+\mathrm{e}=\mathrm{H}_{2} \mathrm{PD}$ & $-0.262 \mathrm{~V}$ & $10 \mathrm{~cm} / \mathrm{s}$ \\
\hline E2 & $\mathrm{H}_{2} \mathrm{PD}^{2+}+\mathrm{e}=\mathrm{H}_{2} \mathrm{PD}^{+}$ & $+0.256 \mathrm{~V}$ & $10 \mathrm{~cm} / \mathrm{s}$ \\
\hline E4 & $\mathrm{HPD}^{+}+\mathrm{e}=\mathrm{HPD}$ & $-0.362 \mathrm{~V}$ & $10 \mathrm{~cm} / \mathrm{s}$ \\
\hline PT1 & $\mathrm{H}_{2} \mathrm{PD}^{+}+\mathrm{B}=\mathrm{HPD}+\mathrm{HB}^{+}$ & $\left(1.90 \times 10^{-9}\right)=\mathrm{K}_{\mathrm{rad}}$ & $1.90 \mathrm{M}^{-1} \mathrm{~s}^{-1}$ \\
\hline PT2 & $\mathrm{H}_{2} \mathrm{PD}^{2+}+\mathrm{B}=\mathrm{HPD}^{+}+\mathrm{HB}^{+}$ & $52.8=\mathrm{K}_{\mathrm{ox}}$ & $1.0 \times 10^{9} \mathrm{M}^{-1} \mathrm{~s}^{-1}$ \\
\hline
\end{tabular}

${ }^{1} \alpha=0.5$ for all heterogeneous electron transfers; electrode area $=0.0206 \mathrm{~cm}^{2} ; \mathrm{Ru}=336 \Omega$; D of all species $=$ $1.45 \times 10^{5} \mathrm{~cm}^{2} \mathrm{~s}^{-1}$.

${ }^{2}$ Values in parentheses are automatically set by other thermodynamic parameters. 
Table S4. Mechanism and parameters ${ }^{1}$ used for simulating CVs in Figure 8a (Concerted ProtonElectron Transfer square scheme).

\begin{tabular}{|l|l|l|l|}
\hline & Reaction & $\mathbf{E}^{\circ}$ or $\mathbf{K}^{2}$ & $\mathbf{k}^{\circ}$ or kf \\
\hline E1 & $\mathrm{H}_{2} \mathrm{PD}^{+}+\mathrm{e}=\mathrm{H}_{2} \mathrm{PD}$ & $-0.262 \mathrm{~V}$ & $10 \mathrm{~cm} / \mathrm{s}$ \\
\hline E2 & $\mathrm{H}_{2} \mathrm{PD}^{2+}+\mathrm{e}=\mathrm{H}_{2} \mathrm{PD}^{+}$ & $+0.256 \mathrm{~V}$ & $10 \mathrm{~cm} / \mathrm{s}$ \\
\hline E3 & $\mathrm{HPD}^{+}+\mathrm{HB}^{+}+\mathrm{e}=\mathrm{H}_{2} \mathrm{PD}^{+}+\mathrm{B}$ & $+0.154 \mathrm{~V}$ & $10 \mathrm{~cm} / \mathrm{s}$ \\
\hline E4 & $\mathrm{HPD}^{+}+\mathrm{e}=\mathrm{HPD}$ & $-0.362 \mathrm{~V}$ & $10 \mathrm{~cm} / \mathrm{s}$ \\
\hline PT1 & $\mathrm{H}_{2} \mathrm{PD}^{+}+\mathrm{B}=\mathrm{HPD}+\mathrm{HB}^{+}$ & $\left(1.90 \times 10^{-9}\right)=\mathrm{K}_{\mathrm{rad}}$ & $1.90 \mathrm{M}^{-1} \mathrm{~s}^{-1}$ \\
\hline PT2 & $\mathrm{H}_{2} \mathrm{PD}^{2+}+\mathrm{B}=\mathrm{HPD}^{+}+\mathrm{HB}^{+}$ & $(52.8)=\mathrm{K}_{\mathrm{ox}}$ & $1.0 \times 10^{9} \mathrm{M}^{-1} \mathrm{~s}^{-1}$ \\
\hline
\end{tabular}

${ }^{1} \alpha=0.5$ for all heterogeneous electron transfers; electrode area $=0.0206 \mathrm{~cm}^{2} ; \mathrm{Ru}=336 \Omega$; D of all species $=$ $1.45 \times 10^{5} \mathrm{~cm}^{2} \mathrm{~s}^{-1}$.

${ }^{2}$ Values in parentheses are automatically set by other thermodynamic parameters.

Table S5. Mechanism and parameters ${ }^{1}$ used for simulating CVs in Figure 8b (Wedge scheme).

\begin{tabular}{|l|l|l|l|}
\hline & Reaction & $\mathbf{E}^{\circ}$ or $\mathbf{K}^{2}$ & $\mathbf{k}^{\circ}$ or kf \\
\hline E1 & $\mathrm{H}_{2} \mathrm{PD}^{+}+\mathrm{e}=\mathrm{H}_{2} \mathrm{PD}$ & $-0.262 \mathrm{~V}$ & $10 \mathrm{~cm} / \mathrm{s}$ \\
\hline E2 & $\mathrm{H}_{2} \mathrm{PD}^{2+}+\mathrm{e}=\mathrm{H}_{2} \mathrm{PD}^{+}$ & $+0.256 \mathrm{~V}$ & $10 \mathrm{~cm} / \mathrm{s}$ \\
\hline E4 & $\mathrm{HPD}^{+}+\mathrm{e}=\mathrm{HPD}$ & $-0.362 \mathrm{~V}$ & $10 \mathrm{~cm} / \mathrm{s}$ \\
\hline E5 & $\mathrm{BH}_{2} \mathrm{PD}^{2+}+\mathrm{e}=\mathrm{BH}_{2} \mathrm{PD}^{+}$ & $(-0.0238 \mathrm{~V})$ & $10 \mathrm{~cm} / \mathrm{s}$ \\
\hline PT1 & $\mathrm{H}_{2} \mathrm{PD}^{+}+\mathrm{B}=\mathrm{HPD}+\mathrm{HB}^{+}$ & $\left(1.90 \times 10^{-9}\right)=\mathrm{K}_{\mathrm{rad}}$ & $1.90 \mathrm{M}^{-1} \mathrm{~s}^{-1}$ \\
\hline PT2 & $\mathrm{H}_{2} \mathrm{PD}^{2+}+\mathrm{B}=\mathrm{HPD}^{+}+\mathrm{HB}^{+}$ & $52.8=\mathrm{K}_{\mathrm{ox}}$ & $1.0 \times 10^{9} \mathrm{M}^{-1} \mathrm{~s}^{-1}$ \\
\hline $\mathrm{HB} 1$ & $\mathrm{H}_{2} \mathrm{PD}^{+}+\mathrm{B}=\mathrm{BH}_{2} \mathrm{PD}^{+}$ & $0.010 \mathrm{M}^{-1}$ & $1.0 \times 10^{7} \mathrm{M}^{-1} \mathrm{~s}^{-1}$ \\
\hline $\mathrm{HB} 2$ & $\mathrm{BH}_{2} \mathrm{PD}^{+}=\mathrm{HPD}+\mathrm{HB}^{+}$ & $\left(1.90 \times 10^{-7} \mathrm{M}\right)$ & $190 \mathrm{~s}^{-1}$ \\
\hline $\mathrm{HB} 3$ & $\mathrm{H}_{2} \mathrm{PD}^{2+}+\mathrm{B}=\mathrm{BH}_{2} \mathrm{PD}^{2+}$ & $\left(528 \mathrm{M}^{-1}\right)$ & $1.0 \times 10^{9} \mathrm{M}^{-1} \mathrm{~s}^{-1}$ \\
\hline $\mathrm{HB} 4$ & $\mathrm{BH}_{2} \mathrm{PD}^{2+}=\mathrm{HPD}^{+}+\mathrm{HB}^{+}$ & $0.10 \mathrm{M}$ & $1.0 \times 10^{8} \mathrm{~s}^{-1}$ \\
\hline
\end{tabular}

${ }^{1} \alpha=0.5$ for all heterogeneous electron transfers; electrode area $=0.0206 \mathrm{~cm}^{2} ; \mathrm{Ru}=336 \Omega$; $\mathrm{D}$ of all species $=$ $1.45 \times 10^{5} \mathrm{~cm}^{2} \mathrm{~s}^{-1}$.

${ }^{2}$ Values in parentheses are automatically set by other thermodynamic parameters. 

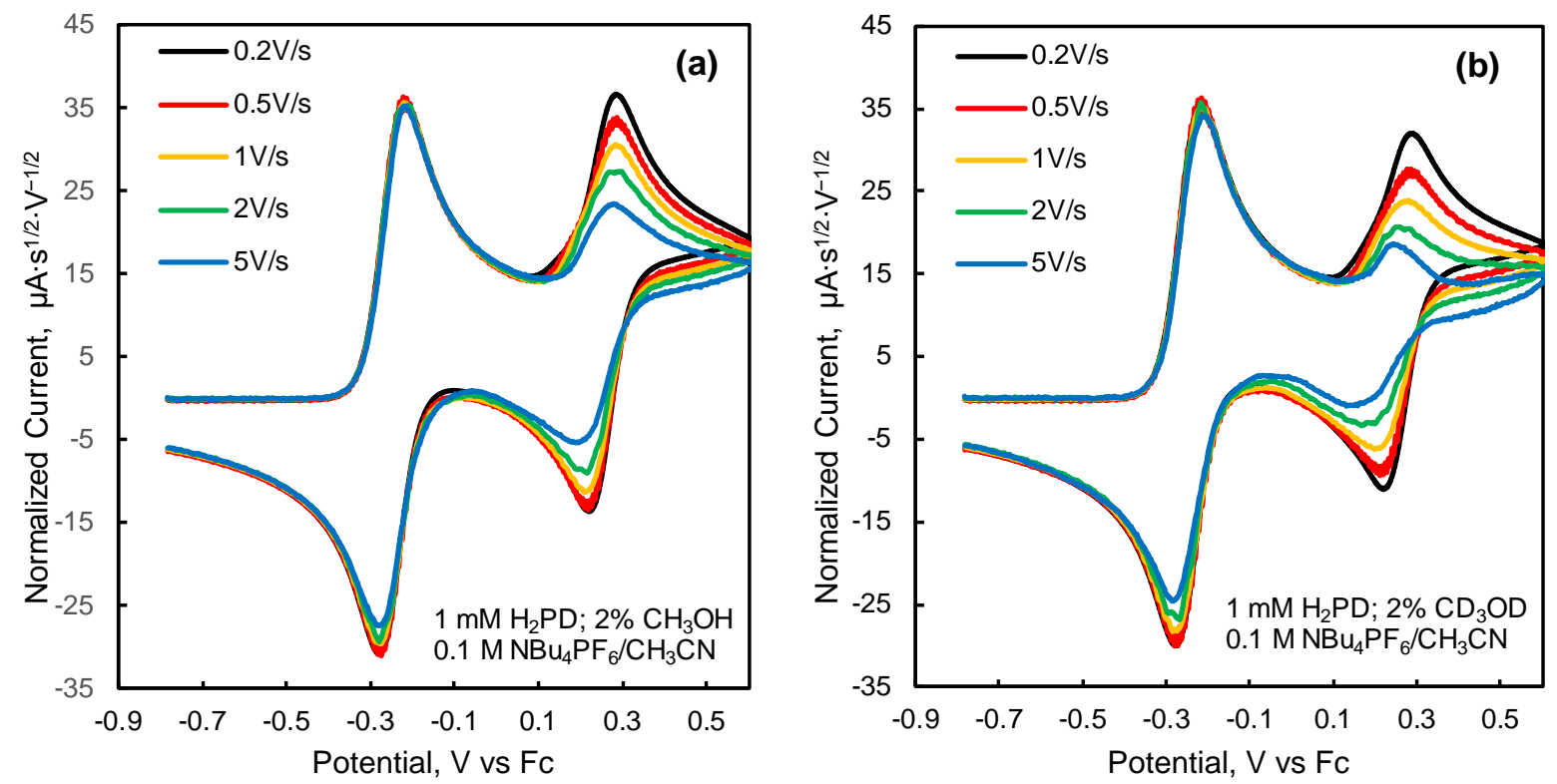

Figure S8. Background-subtracted CV's of $1 \mathrm{mM} \mathrm{H} \mathrm{H}_{2} \mathrm{PD}$ in $0.1 \mathrm{M} \mathrm{NBu}_{4} \mathrm{PF}_{6} / \mathrm{CH}_{3} \mathrm{CN}$ at different scan rates containing either $2 \%$ anhydrous (a) $\mathrm{CH}_{3} \mathrm{OH}$ or (b) $\mathrm{CD}_{3} \mathrm{OD}$. The current has been normalized by dividing by the square root of the scan rate.
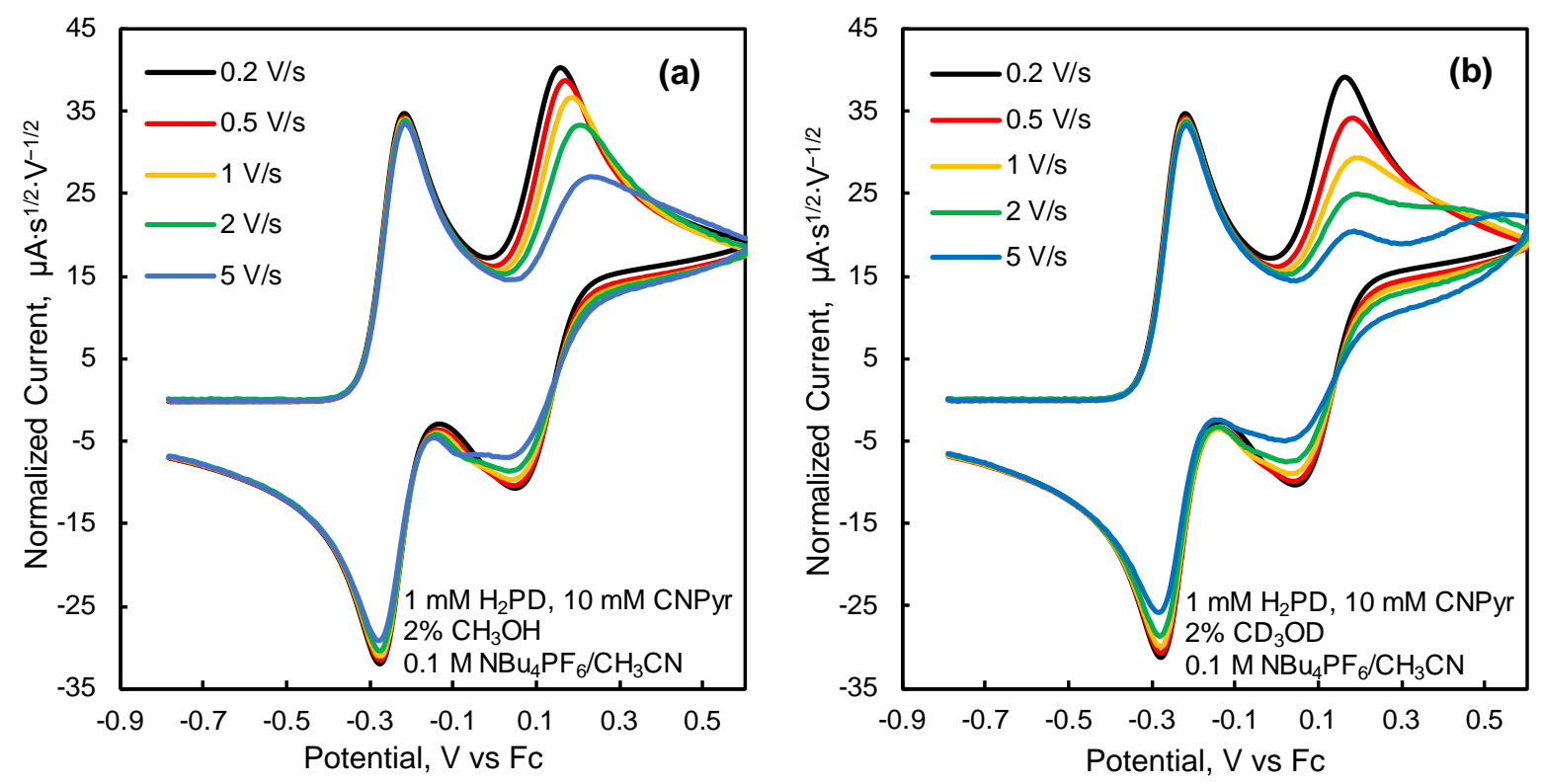

Figure S9. Background-subtracted CV's of $1 \mathrm{mM} \mathrm{H} \mathrm{H}_{2} \mathrm{PD}+10 \mathrm{mM}$ 4-cyanopyridine in $0.1 \mathrm{M}$ $\mathrm{NBu}_{4} \mathrm{PF}_{6} / \mathrm{CH}_{3} \mathrm{CN}$ at different scan rates containing either $2 \%$ anhydrous (a) $\mathrm{CH}_{3} \mathrm{OH}$ or (b) $\mathrm{CD}_{3} \mathrm{OD}$. The current has been normalized by dividing by the square root of the scan rate. 

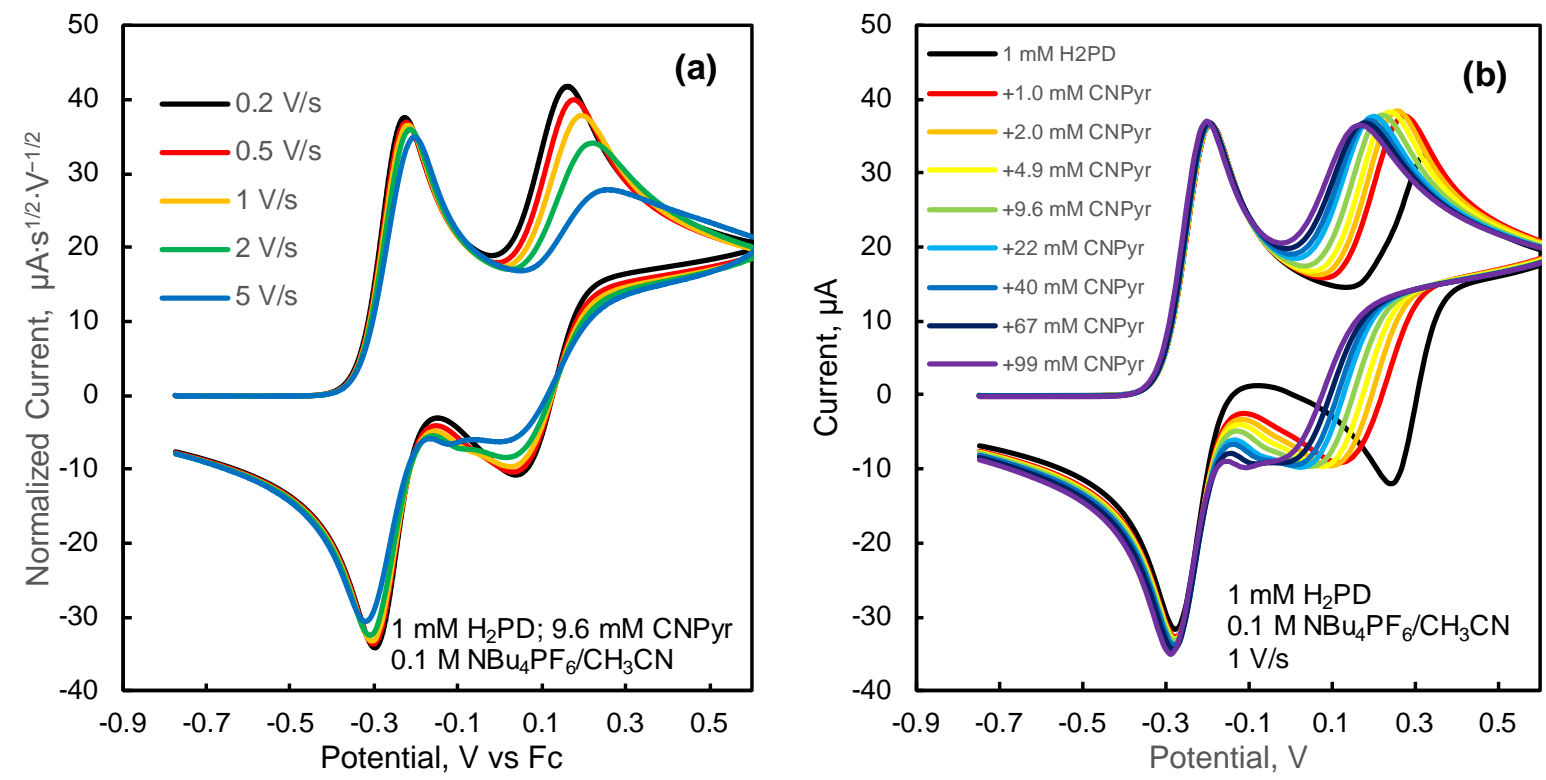

Figure S10. Background-subtracted CV's of $1 \mathrm{mM} \mathrm{H}_{2} \mathrm{PD}$ in $0.1 \mathrm{M} \mathrm{NBu}_{4} \mathrm{PF}_{6} / \mathrm{CH}_{3} \mathrm{CN}$ at (a) different scan rates with $9.6 \mathrm{mM}$ 4-cyanopyridine, and (b) at $1 \mathrm{~V} / \mathrm{s}$ with different concentrations of 4-cyanopyridine. The current in (a) has been normalized by dividing by the square root of the scan rate. 